Pré-Publicações do Departamento de Matemática

Universidade de Coimbra

Preprint Number 04-33

\title{
INTRINSIC SCALING FOR PDE'S WITH AN EXPONENTIAL NONLINEARITY
}

\author{
EURICA HENRIQUES AND JOSÉ MIGUEL URBANO
}

\begin{abstract}
We consider strongly degenerate equations in divergence form of the type

$$
\partial_{t} u-\nabla \cdot\left(|u|^{\gamma(x, t)} \nabla u\right)=f
$$

where the exponential nonlinearity satisfies the condition $0<\gamma^{-} \leq \gamma(x, t) \leq \gamma^{+}$. We show, by means of intrinsic scaling, that weak solutions are locally continuous.

KEYWORDS: porous medium equation, degenerate PDE's, regularity theory, intrinsic scaling.

AMS Subject Classification (2000): 35B65, 35D10, 35K65.
\end{abstract}

\section{Introduction}

Intrinsic scaling has proved to be a remarkably powerful tool in the analysis of the regularity of weak solutions of nonlinear partial differential equations. Devised by DiBenedetto in the early 1980's, it flourished in the last few years with applications to a wide range of nonlinear equations that exhibit some form of singularity or degeneracy, or both (see, e.g., [5], [6], [8] or [10]).

As many fundamental ideas, intrinsic scaling is strikingly easy to grasp: it amounts to analyze each equation in a geometrical framework related to its structure. The precise understanding of this statement requires a knowledge of the seminal work of DeGiorgi and Moser on regularity theory for uniformly elliptic and parabolic PDE's with measurable coefficients, namely the iterative methods they developed. These methods consist on a fine analysis of the behaviour of the oscillation for the solutions of the PDE in a sequence of nested and shrinking cylinders, showing that it converges to zero and, when possible, exactly how this happens. The conclusion is that solutions are continuous and, in many instances, that a modulus of continuity can be derived. In the elliptic case, these results could be extended to equations with the full quasilinear structure but, in the parabolic case, only to equations whose principal part has exactly a linear growth with respect to the modulus of

Received November 12, 2004.

Research supported by CMUC/FCT, Project POCTI/34471/MAT/2000 and PRODEP-FSE 
the gradient of the solution; it appeared that a degeneracy or singularity of the principal part played a peculiar role. The clear understanding of that role had to wait for intrinsic scaling and follows from a single unifying idea: the diffusion processes in the equations evolve in a time scale determined instant by instant by the solution itself, so that, loosely speaking, they can be regarded as the heat equation in their own intrinsic time-configuration. A precise description of this fact, as well as its effectiveness, is closely linked to its technical implementation, namely in terms of the construction of the cylinders in which the iteration process takes place. For a modern account of the theory see [5].

The theory of degenerate and singular elliptic and parabolic equations is one of the branches of modern analysis both in view of the physical significance of the equations at hand and of the novel analytical techniques that they generate. The class of such equations is large, ranging from flows by mean curvature to Monge-Ampére equations to infinity-Laplacian. Parabolic equations in divergence form of the type

$$
\partial_{t} u-\nabla \cdot\left(|u|^{\gamma(x, t)} \nabla u\right)=f \quad \text { in } \quad \mathcal{D}^{\prime}\left(\Omega_{T}\right),
$$

with a variable exponent of nonlinearity $\gamma$, are generalizations of the famous porous medium equation and occur as a model for the flow of electrorheological fluids (cf. [7]). The main feature in equation (1) is clearly the exponential nonlinearity that makes it extremely degenerate. Recently, Antontsev and Shmarev [1] obtained results on the existence and uniqueness of weak solutions of (1), together with some localization properties. Under appropriate assumptions, we prove in this paper that weak solutions are locally continuous. The technique we use is intrinsic scaling.

For the porous medium equation, that corresponds to the case $\gamma \equiv C$, an abundant literature is available. In the case $N>1$, questions of regularity were first considered by Caffarelli and Friedman in [2]. In more generality, DiBenedetto proved in [3] that weak solutions of

$$
\partial_{t} \beta(u)-\nabla \cdot \mathbf{a}(x, t, u, \nabla u)=b(x, t, u, \nabla u),
$$

where $\beta(s)=|s|^{\frac{1}{m}} \operatorname{sign} s, m>1$, are locally continuous imposing no restrictions on the sign of $u$ nor any relationship between the (possibly nonlinear) $\mathbf{a}(x, t, u, \nabla u), b(x, t, u, \nabla u)$ and the graph $\beta$. Other generalizations, to equations with a double degeneracy, were considered in [8] and [10]. 


\section{The equation in its own geometry}

Consider the equation

$$
\partial_{t} u-\nabla \cdot\left(|u|^{\gamma(x, t)} \nabla u\right)=0 \quad \text { in } \quad \mathcal{D}^{\prime}\left(\Omega_{T}\right)
$$

where $\Omega_{T}=\Omega \times(0, T], \Omega$ is a regular bounded domain in $\mathbf{R}^{N}$, and $0<T<$ $\infty$. We assume the exponent $\gamma$ satisfies the following assumptions:

(A1) $\gamma \in L^{\infty}\left(0, T ; W^{1, \infty}(\Omega)\right), \quad$ with $\quad\|\gamma\|_{L^{\infty}\left(0, T ; W^{1, \infty}(\Omega)\right)}=M$

(A2) For constants $\gamma^{-}, \gamma^{+}>0$,

$$
0<\gamma^{-} \leq \gamma(x, t) \leq \gamma^{+}<\infty, \quad \text { a.e. }(x, t) \in \Omega_{T} .
$$

Definition 1. We say that a measurable function $u$ is a local weak solution of (2) if

- $u \in L^{\infty}\left(0, T ; L^{\infty}(\Omega)\right)$ with $u(x, t) \in[0,1]$ a.e. in $\Omega_{T}$;

- $u \in C\left(0, T ; L^{2}(\Omega)\right)$ and $u^{\frac{\gamma(x, t)}{2}} \nabla u \in L^{2}\left(0, T ; L^{2}(\Omega)\right)$;

- for every compact $K \subset \Omega$ and for every subinterval $\left[t_{1}, t_{2}\right] \subset(0, T]$,

$$
\left.\int_{K} u \phi d x\right|_{t_{1}} ^{t_{2}}+\int_{t_{1}}^{t_{2}} \int_{K}\left\{-u \partial_{t} \phi+u^{\gamma(x, t)} \nabla u \cdot \nabla \phi\right\} d x d t=0,
$$

for all functions $\phi \in H_{\mathrm{loc}}^{1}\left(0, T ; L^{2}(K)\right) \cap L_{\mathrm{loc}}^{2}\left(0, T ; H_{0}^{1}(K)\right)$.

It is convenient to our purposes to have at hand a definition of weak solution involving a discrete time derivative. Making use of the Steklov average of a function (see [4] for more details) we obtain the following formulation which is equivalent to $(3)$ :

- for every compact $K \subset \Omega$ and for all $0<t<T-h$,

$$
\int_{K \times\{t\}} \partial_{t} u_{h} \phi d x+\int_{K \times\{t\}}\left(u^{\gamma(x, \cdot)} \nabla u\right)_{h} \cdot \nabla \phi d x=0,
$$

for all $\phi \in H_{0}^{1}(K)$.

In the recent paper [1], it is proved, under less restrictive assumptions on $\gamma$, that there exists a unique solution to the initial boundary value problem associated with this equation and that the solution is bounded. It is also shown that the solution is nonnegative if the initial data is nonnegative; that 
is why the assumption $u(x, t) \in[0,1]$ a.e. in $\Omega_{T}$ included in Definition 1 is reasonable.

Observe that the equation is degenerate at points where $u=0$, since for $\gamma$ verifying $(A 2)$, the diffusion coefficient $u^{\gamma(x, t)}$ vanishes at those points. To deal with this fact we need to define an intrinsic geometric configuration for this specific PDE. We start with some notation. Given $x_{0} \in \mathbf{R}^{N}$, define the $N$-dimensional cube

$$
\left[x_{0}+K_{\rho}\right]:=\left\{x: \max _{1 \leq i \leq N}\left|x_{i}-x_{0 i}\right|<\rho\right\} .
$$

Given the pair $\left(x_{0}, t_{0}\right) \in \mathbf{R}^{N+1}$, define the cylinder

$$
\left[\left(x_{0}, t_{0}\right)+Q(\tau, \rho)\right]:=\left[x_{0}+K_{\rho}\right] \times\left(t_{0}-\tau, t_{0}\right) .
$$

Now, let $\left(x_{0}, t_{0}\right)$ be a point of the space-time domain $\Omega_{T}$ that, by translation, we may assume to be $(0,0)$. Consider small positive numbers $\epsilon>0$ and $R>0$ such that the cylinder $Q\left(R^{2-\epsilon}, R\right) \subset \Omega_{T}$ and define

$$
\mu^{-}:=\operatorname{ess}_{Q\left(R^{2-\epsilon}, R\right)} u ; \quad \mu^{+}:=\operatorname{ess}_{Q\left(R^{2-\epsilon}, R\right)}^{\operatorname{eup}} u ; \quad \omega:=\underset{Q\left(R^{2-\epsilon}, R\right)}{\operatorname{ess} \text { osc }} u=\mu^{+}-\mu^{-} .
$$

Recalling that (2) is degenerate at the points where $u=0$, the interesting case to investigate is when $\mu^{-}=0$ and, consequently, $\mu^{+}=\omega$. From now on, we will assume this is in force.

Construct the cylinder

$$
Q\left(a_{0} R^{2}, R\right), \quad a_{0}=\left(\frac{4}{\omega}\right)^{\gamma^{+}},
$$

and assume that

$$
\omega \geq 4 R^{\frac{\epsilon}{\gamma^{+}}}
$$

This implies that $Q\left(a_{0} R^{2}, R\right) \subset Q\left(R^{2-\epsilon}, R\right)$ and then

$$
\underset{Q\left(a_{0} R^{2}, R\right)}{\operatorname{ess} \text { osc }} u \leq \omega .
$$

For technical reasons, we make the extra assumption that $\epsilon \leq 2 \gamma^{+}$.

Remark 1. If (5) does not hold, then the oscillation $\omega$ goes to zero when the radius $R$ goes to zero, in a way given by the reverse inequality, and there is nothing to prove. Note that, in general, (6) is not verified a priori for a given cylinder. The cylinder dimensions had to be defined in terms of the oscillation of the solution within it. 
When $\gamma \equiv 0, a_{0}=1$ and we recover the standard parabolic cylinder with the natural homogeneity of the space and time variables.

The proof of the main result of this paper relies on the study of two complementary cases that can be described as follows: either $u$ is essentially away from its infimum in the constructed cylinder, or this does not hold. In either case, we are able to reduce the oscillation of $u$ within a smaller cylinder with the same "vertex". The alternative can be formulated as

Given $\nu_{0} \in(0,1)$, to be determined in terms of the data and $\omega$, either

$$
\left|(x, t) \in Q\left(a_{0} R^{2}, R\right): u(x, t)<\frac{\omega}{2}\right| \leq \nu_{0}\left|Q\left(a_{0} R^{2}, R\right)\right|
$$

or, noting that $\mu^{+}-\frac{\omega}{2}=\frac{\omega}{2}$,

$$
\left|(x, t) \in Q\left(a_{0} R^{2}, R\right): u(x, t)>\mu^{+}-\frac{\omega}{2}\right|<\left(1-\nu_{0}\right)\left|Q\left(a_{0} R^{2}, R\right)\right| .
$$

The analysis of this alternative leads to the following result.

Proposition 1. There exist positive numbers $\nu_{0}, \sigma \in(0,1)$, depending on the data and on $\omega$, such that

$$
\underset{Q\left(\frac{\nu_{0}}{2} a_{0}\left(\frac{R}{2}\right)^{2}, \frac{R}{2}\right)}{\operatorname{ess} \text { osc }} \quad u \leq \sigma \omega
$$

An immediate consequence is

Theorem 1. Under assumptions $(A 1)-(A 2)$ any locally bounded weak solution of (2) is locally continuous in $\Omega_{T}$.

The proof of Theorem 1 is a (now standard) consequence of Proposition 1. Indeed, from (9) one can recursively define a sequence $Q_{n}$ of nested and shrinking cylinders and a sequence $\omega_{n}$ converging to zero, such that

$$
\underset{Q_{n}}{\operatorname{ess}} \operatorname{OSc} u \leq \omega_{n} \text {. }
$$

Continuity of $u$ follows. For a proof of these facts, the reader is invited to read the survey paper [5]. 
Remark 2. We stress that we only obtain continuity of $u$, and are unable to derive a modulus, since the constant $\sigma$ appearing in Proposition 1 depends on the oscillation $\omega$. See [9] for the details.

\section{Reducing the oscillation}

Assume that (7) is verified. In the following, we determine the number $\nu_{0}$ and guarantee that the solution $u$ is above a smaller level within a smaller cylinder.

Proposition 2. There exists $\nu_{0} \in(0,1)$, depending only on the data and $\omega$, such that if (7) holds true then

$$
u(x, t) \geq \frac{\omega}{4}, \quad \text { a.e. }(x, t) \in Q\left(a_{0}\left(\frac{R}{2}\right)^{2}, \frac{R}{2}\right) .
$$

Proof. Define two decreasing sequences of positive numbers

$$
R_{n}=\frac{R}{2}+\frac{R}{2^{n+1}}, \quad k_{n}=\frac{\omega}{4}+\frac{\omega}{2^{n+2}}, \quad n=0,1, \ldots
$$

and construct the family of nested and shrinking cylinders $Q_{n}=Q\left(a_{0} R_{n}^{2}, R_{n}\right)$.

Introduce the function $u_{\omega}=\max \left\{u, \frac{\omega}{4}\right\}$. In the weak formulation (4) take $\phi=-\left(\left(u_{\omega}\right)_{h}-k_{n}\right)_{-} \xi_{n}^{2}$, where $0 \leq \xi_{n} \leq 1$ are smooth cutoff functions defined in $Q_{n}$ and satisfying

$$
\left\{\begin{array}{l}
\xi_{n} \equiv 1 \quad \text { in } \quad Q_{n+1}, \quad \xi_{n} \equiv 0 \quad \text { on the parabolic boundary of } Q_{n} \\
\left|\nabla \xi_{n}\right| \leq \frac{2^{n+2}}{R}, \quad\left|\Delta \xi_{n}\right| \leq \frac{2^{2(n+2)}}{R^{2}}, \quad 0<\partial_{t} \xi_{n} \leq \frac{2^{2(n+2)}}{a_{0} R^{2}}
\end{array}\right.
$$

and integrate in time over $\left(-a_{0} R_{n}^{2}, t\right)$, for $t \in\left(-a_{0} R_{n}^{2}, 0\right)$. We obtain (omitting the $d x$ and $d t$ in all integrals from now on)

$$
\begin{gathered}
I_{1}+I_{2}:=\int_{-a_{0} R_{n}^{2}}^{t} \int_{K_{R_{n}}} \partial_{t} u_{h}\left[-\left(\left(u_{\omega}\right)_{h}-k_{n}\right)_{-} \xi_{n}^{2}\right] \\
+\int_{-a_{0} R_{n}^{2}}^{t} \int_{K_{R_{n}}}\left(u^{\gamma} \nabla u\right)_{h} \cdot \nabla\left[-\left(\left(u_{\omega}\right)_{h}-k_{n}\right)_{-} \xi_{n}^{2}\right]=0 .
\end{gathered}
$$

Concerning the first integral, we have

$$
I_{1}=\int_{-a_{0} R_{n}^{2}}^{t} \int_{K_{R_{n}}} \partial_{t} u_{h}\left[-\left(\left(u_{\omega}\right)_{h}-k_{n}\right)_{-} \xi_{n}^{2}\right] \chi_{\left[\left(u_{\omega}\right)_{h}=u_{h}\right]}
$$




$$
\begin{aligned}
& +\int_{-a_{0} R_{n}^{2}}^{t} \int_{K_{R_{n}}} \partial_{t} u_{h}\left[-\left(\left(u_{\omega}\right)_{h}-k_{n}\right)_{-} \xi_{n}^{2}\right] \chi_{\left[\left(u_{\omega}\right)_{h}=\frac{\omega}{4}\right]} \\
= & \frac{1}{2} \int_{-a_{0} R_{n}^{2}}^{t} \int_{K_{R_{n}}} \partial_{t}\left[\left(\left(u_{\omega}\right)_{h}-k_{n}\right)_{-}^{2}\right] \xi_{n}^{2} \\
& +\left(\frac{\omega}{2^{n+2}}\right) \int_{-a_{0} R_{n}^{2}}^{t} \int_{K_{R_{n}}} \partial_{t}\left[\left(u_{h}-\frac{\omega}{4}\right)_{-}\right] \xi_{n}^{2} .
\end{aligned}
$$

Next, we integrate by parts and let $h \rightarrow 0$. Using Lemma 3.2 of Chapter I of [4] we get

$$
\begin{gathered}
\frac{1}{2} \int_{K_{R_{n}} \times\{t\}}\left(u_{\omega}-k_{n}\right)_{-}^{2} \xi_{n}^{2}-\int_{-a_{0} R_{n}^{2}}^{t} \int_{K_{R_{n}}}\left(u_{\omega}-k_{n}\right)_{-}^{2} \xi_{n} \partial_{t} \xi_{n} \\
+\left(\frac{\omega}{2^{n+2}}\right) \int_{K_{R_{n} \times\{t\}}}\left(u-\frac{\omega}{4}\right)_{-} \xi_{n}^{2}-2\left(\frac{\omega}{2^{n+2}}\right) \int_{-a_{0} R_{n}^{2}}^{t} \int_{K_{R_{n}}}\left(u-\frac{\omega}{4}\right)_{-} \xi_{n} \partial_{t} \xi_{n} \\
\geq \frac{1}{2} \int_{K_{R_{n}} \times\{t\}}\left(u_{\omega}-k_{n}\right)_{-}^{2} \xi_{n}^{2}-3\left(\frac{\omega}{4}\right)^{2} \frac{2^{2(n+2)}}{a_{0} R^{2}} \int_{-a_{0} R_{n}^{2}}^{t} \int_{K_{R_{n}}} \chi_{\left[u_{\omega} \leq k_{n}\right]}
\end{gathered}
$$

since the third term is nonnegative and, for $0 \leq u \leq \frac{\omega}{4}$,

$$
u_{\omega}=\frac{\omega}{4} \leq k_{n}
$$

and, for $\frac{\omega}{4}<u=u_{\omega} \leq k_{n}$,

$$
\left(u_{\omega}-k_{n}\right)_{-} \leq k_{n}-u_{\omega}=k_{n}-u<k_{n}-\frac{\omega}{4}=\frac{\omega}{2^{n+2}} \leq \frac{\omega}{4} .
$$

Concerning $I_{2}$, we first pass to the limit in $h$ to get

$$
\begin{aligned}
I_{2} \rightarrow & \int_{-a_{0} R_{n}^{2}}^{t} \int_{K_{R_{n}}} u^{\gamma} \nabla u \cdot \nabla\left(-\left(u_{\omega}-k_{n}\right)_{-} \xi_{n}^{2}\right) \\
= & \int_{-a_{0} R_{n}^{2}}^{t} \int_{K_{R_{n}}} u^{\gamma} \nabla u \cdot \nabla\left(-\left(u_{\omega}-k_{n}\right)_{-} \xi_{n}^{2}\right) \chi_{\left[u_{\omega}=u\right]} \\
& +\int_{-a_{0} R_{n}^{2}}^{t} \int_{K_{R_{n}}} u^{\gamma} \nabla u \cdot \nabla\left(-\left(u_{\omega}-k_{n}\right)_{-} \xi_{n}^{2}\right) \chi_{\left[u_{\omega}=\frac{\omega}{4}\right]} \\
= & \int_{-a_{0} R_{n}^{2}}^{t} \int_{K_{R_{n}}} u_{\omega}^{\gamma}\left|\nabla\left(u_{\omega}-k_{n}\right)_{-}\right|^{2} \xi_{n}^{2}
\end{aligned}
$$




$$
\begin{aligned}
& +2 \int_{-a_{0} R_{n}^{2}}^{t} \int_{K_{R_{n}}} u_{\omega}^{\gamma} \nabla\left(u_{\omega}-k_{n}\right)_{-} \cdot \nabla \xi_{n} \xi_{n}\left(u_{\omega}-k_{n}\right)_{-} \\
& +2\left(\frac{\omega}{2^{n+2}}\right) \int_{-a_{0} R_{n}^{2}}^{t} \int_{K_{R_{n}}}-u^{\gamma} \nabla u \cdot \nabla \xi_{n} \xi_{n} \chi_{\left[u \leq \frac{\omega}{4}\right]} \\
& \geq \frac{1}{2} \int_{-a_{0} R_{n}^{2}}^{t} \int_{K_{R_{n}}} u_{\omega}^{\gamma}\left|\nabla\left(u_{\omega}-k_{n}\right)_{-}\right|^{2} \xi_{n}^{2} \\
& -2 \int_{-a_{0} R_{n}^{2}}^{t} \int_{K_{R_{n}}} u_{\omega}^{\gamma}\left|\nabla \xi_{n}\right|^{2}\left(u_{\omega}-k_{n}\right)_{-}^{2} \\
& +2\left(\frac{\omega}{2^{n+2}}\right) \int_{-a_{0} R_{n}^{2}}^{t} \int_{K_{R_{n}}} \nabla\left(\int_{u}^{\frac{\omega}{4}} s^{\gamma} d s\right) \cdot \nabla \xi_{n} \xi_{n} \chi_{\left[u \leq \frac{\omega}{4}\right]} \\
& +2\left(\frac{\omega}{2^{n+2}}\right) \int_{-a_{0} R_{n}^{2}}^{t} \int_{K_{R_{n}}}\left(\int_{u}^{\frac{\omega}{4}}(-\ln s) s^{\gamma} d s\right) \nabla \gamma \cdot \nabla \xi_{n} \xi_{n} \chi_{\left[u \leq \frac{\omega}{4}\right]} \\
& \geq \frac{1}{2} \int_{-a_{0} R_{n}^{2}}^{t} \int_{K_{R_{n}}} u_{\omega}^{\gamma}\left|\nabla\left(u_{\omega}-k_{n}\right)_{-}\right|^{2} \xi_{n}^{2} \\
& -2 \int_{-a_{0} R_{n}^{2}}^{t} \int_{K_{R_{n}}} u_{\omega}^{\gamma}\left|\nabla \xi_{n}\right|^{2}\left(u_{\omega}-k_{n}\right)_{-}^{2} \\
& -2\left(\frac{\omega}{2^{n+2}}\right) \int_{-a_{0} R_{n}^{2}}^{t} \int_{K_{R_{n}}}\left(\int_{u}^{\frac{\omega}{4}} s^{\gamma} d s\right)\left(\xi_{n}\left|\Delta \xi_{n}\right|+\left|\nabla \xi_{n}\right|^{2}\right) \chi_{\left[u \leq \frac{\omega}{4}\right]} \\
& -\left(\frac{\omega}{2^{n+2}}\right) \int_{-a_{0} R_{n}^{2}}^{t} \int_{K_{R_{n}}}\left(\int_{u}^{\frac{\omega}{4}}(-\ln s) s^{\gamma} d s\right)|\nabla \gamma|^{2} \xi_{n} \chi_{\left[u \leq \frac{\omega}{4}\right]} \\
& -\left(\frac{\omega}{2^{n+2}}\right) \int_{-a_{0} R_{n}^{2}}^{t} \int_{K_{R_{n}}}\left(\int_{u}^{\frac{\omega}{4}}(-\ln s) s^{\gamma} d s\right)\left|\nabla \xi_{n}\right|^{2} \xi_{n} \chi_{\left[u \leq \frac{\omega}{4}\right]} \\
& =: I_{2}^{\prime} \text {. }
\end{aligned}
$$

The first inequality is obtained by means of Cauchy's inequality with $\epsilon=\frac{1}{4}$, and the observation that, within the set $\left[u \leq \frac{\omega}{4}\right]$,

$$
\nabla\left(\int_{u}^{\frac{\omega}{4}} s^{\gamma} d s\right)=-u^{\gamma} \nabla u-\left(\int_{u}^{\frac{\omega}{4}}(-\ln s) s^{\gamma} d s\right) \nabla \gamma
$$

The second inequality is a consequence of integration by parts and again Cauchy's inequality, this time with $\epsilon=\frac{1}{2}$. 
Next, observe that for $\frac{\omega}{4}<u=u_{\omega} \leq k_{n}$, we get

$$
\begin{gathered}
\left(u_{\omega}-k_{n}\right)_{-}=k_{n}-u_{\omega}=k_{n}-u<\frac{\omega}{2^{n+2}} \leq \frac{\omega}{4} ; \\
\frac{1}{a_{0}} \leq\left(\frac{\omega}{4}\right)^{\gamma}<u_{\omega}^{\gamma} \leq 1 \quad \text { using } \quad(A 2),
\end{gathered}
$$

and for $u \leq \frac{\omega}{4}$, we get

$$
\begin{gathered}
u_{\omega}=\frac{\omega}{4} \leq k_{n} ; \\
\int_{u}^{\frac{\omega}{4}} s^{\gamma} d s \leq\left(\frac{\omega}{4}\right)^{\gamma}\left(\frac{\omega}{4}-u\right) \leq \frac{\omega}{4} ; \\
\int_{u}^{\frac{\omega}{4}}(-\ln s) s^{\gamma} d s \leq\left(\frac{\omega}{4}\right)^{\gamma} \int_{u}^{\frac{\omega}{4}}(-\ln s) d s \leq \int_{u}^{\frac{\omega}{4}}(-\ln s) d s \\
=-\frac{\omega}{4} \ln \left(\frac{\omega}{4}\right)+u \ln u+\frac{\omega}{4}-u \leq\left(\frac{\omega}{4}-u\right)\left\{\ln \left(\frac{4}{\omega}\right)+1\right\} \leq\left(\frac{\omega}{4}\right) \frac{4}{\omega}=1 .
\end{gathered}
$$

Using these inequalities, recalling the conditions on $\xi_{n}$, the fact that $\omega \leq 1$, and the following consequence of (5) and the choice $\epsilon \leq 2 \gamma^{+}$

$$
\frac{1}{R^{2}} \geq \frac{\frac{\omega}{4}}{R^{\frac{\epsilon}{\gamma^{+}}}} \geq 1
$$

$I_{2}^{\prime}$ is bounded from below by

$$
\begin{gathered}
\frac{1}{2 a_{0}} \int_{-a_{0} R_{n}^{2}}^{t} \int_{K_{R_{n}}}\left|\nabla\left(u_{\omega}-k_{n}\right)_{-}\right|^{2} \xi_{n}^{2} \\
-\frac{C(M)}{\omega}\left(\frac{\omega}{4}\right)^{2} \frac{2^{2(n+2)}}{R^{2}} \int_{-a_{0} R_{n}^{2}}^{t} \int_{K_{R_{n}}} \chi_{\left[u_{\omega} \leq k_{n}\right]} .
\end{gathered}
$$

Combining the above results, we obtain the energy estimates

$$
\begin{gathered}
\sup _{-a_{0} R_{n}^{2}<t<0} \int_{K_{R_{n}} \times\{t\}}\left(u_{\omega}-k_{n}\right)_{-}^{2} \xi_{n}^{2}+\frac{1}{a_{0}} \int_{-a_{0} R_{n}^{2}}^{0} \int_{K_{R_{n}}}\left|\nabla\left(u_{\omega}-k_{n}\right)_{-}\right|^{2} \xi_{n}^{2} \\
\leq 6\left(\frac{\omega}{4}\right)^{2} \frac{2^{2(n+2)}}{a_{0} R^{2}} \int_{-a_{0} R_{n}^{2}}^{0} \int_{K_{R_{n}}} \chi_{\left[u_{\omega} \leq k_{n}\right]} \\
+\frac{C(M)}{\omega}\left(\frac{\omega}{4}\right)^{2} \frac{2^{2(n+2)}}{R^{2}} \int_{-a_{0} R_{n}^{2}}^{0} \int_{K_{R_{n}}} \chi_{\left[u_{\omega} \leq k_{n}\right]} .
\end{gathered}
$$


Let us now consider the change of variables

$$
z=\frac{t}{a_{0}}
$$

and define the new functions

$$
\bar{u}_{\omega}(x, z)=u_{\omega}\left(x, a_{0} z\right) \quad ; \quad \bar{\xi}_{n}(x, z)=\xi_{n}\left(x, a_{0} z\right) .
$$

Then the above estimates read

$$
\begin{aligned}
\sup _{-R_{n}^{2}<z<0} & \int_{K_{R_{n}} \times\{z\}}\left(\bar{u}_{\omega}-k_{n}\right)_{-}^{2} \bar{\xi}_{n}^{2}+\int_{-R_{n}^{2}}^{0} \int_{K_{R_{n}}}\left|\nabla\left(\bar{u}_{\omega}-k_{n}\right)_{-}\right|^{2} \bar{\xi}_{n}^{2} \\
\leq & 6\left(\frac{\omega}{4}\right)^{2} \frac{2^{2(n+2)}}{R^{2}} \int_{-R_{n}^{2}}^{0} \int_{K_{R_{n}}} \chi_{\left[\bar{u}_{\omega} \leq k_{n}\right]} \\
& +\frac{C\left(M, \gamma^{+}\right)}{\omega^{1+\gamma^{+}}}\left(\frac{\omega}{4}\right)^{2} \frac{2^{2(n+2)}}{R^{2}} \int_{-R_{n}^{2}}^{0} \int_{K_{R_{n}}} \chi_{\left[\bar{u}_{\omega} \leq k_{n}\right]} \\
\leq & \frac{C\left(M, \gamma^{+}\right)}{\omega^{1+\gamma^{+}}}\left(\frac{\omega}{4}\right)^{2} \frac{2^{2(n+2)}}{R^{2}} A_{n}
\end{aligned}
$$

for $A_{n} \equiv \int_{-R_{n}^{2}}^{0} \int_{K_{R_{n}}} \chi_{\left[\bar{u}_{\omega} \leq k_{n}\right]}$. These estimates imply the inequality

$$
\left\|\left(\bar{u}_{\omega}-k_{n}\right)_{-}\right\|_{V^{2}\left(Q\left(R_{n+1}^{2}, R_{n+1}\right)\right)}^{2} \leq \frac{C\left(M, \gamma^{+}\right)}{\omega^{1+\gamma^{+}}}\left(\frac{\omega}{4}\right)^{2} \frac{2^{2(n+2)}}{R^{2}} A_{n},
$$

where $V^{2}=L^{\infty}\left(L^{2}\right) \cap L^{2}\left(H^{1}\right)$. Using Corollary 3.1 of [4, page 9$]$, we get

$$
\begin{gathered}
\left(\frac{\omega}{4}\right)^{2} \frac{1}{2^{2(n+1)}} A_{n+1}=\left(k_{n}-k_{n+1}\right)^{2} A_{n+1} \leq \iint_{Q\left(R_{n+1}^{2}, R_{n+1}\right)}\left(\bar{u}_{\omega}-k_{n}\right)_{-}^{2} \\
\leq C(N)\left|\left[\bar{u}_{\omega} \leq k_{n}\right] \cap Q\left(R_{n+1}^{2}, R_{n+1}\right)\right|^{\frac{2}{N+2}}\left\|\left(\bar{u}-k_{n}\right)_{-}\right\|_{V^{2}\left(Q\left(R_{n+1}^{2}, R_{n+1}\right)\right)}^{2} \\
\leq C(N) A_{n^{\frac{2}{N+2}}}\left\|\left(\bar{u}-k_{n}\right)_{-}\right\|_{V^{2}\left(Q\left(R_{n+1}^{2}, R_{n+1}\right)\right)}^{2},
\end{gathered}
$$

and consequently

$$
A_{n+1} \leq \frac{C\left(M, N, \gamma^{+}\right)}{\omega^{1+\gamma^{+}}} \frac{2^{4 n}}{R^{2}} A_{n}^{1+\frac{2}{N+2}}
$$


Defining $Y_{n} \equiv \frac{A_{n}}{\left|Q\left(R_{n}^{2}, R_{n}\right)\right|}$ and noting that $\frac{\left|Q\left(R_{n}^{2}, R_{n}\right)\right|^{1+\frac{2}{N+2}}}{\left|Q\left(R_{n+1}^{2}, R_{n+1}\right)\right|} \leq 2^{N+4} R^{2}$, we arrive at the algebraic inequality

$$
Y_{n+1} \leq \frac{C\left(M, N, \gamma^{+}\right)}{\omega^{1+\gamma^{+}}} 2^{4 n} Y_{n}^{1+\frac{2}{N+2}} .
$$

Now, by Lemma 4.1 of [4, page 12], if

$$
Y_{0} \leq C\left(M, N, \gamma^{+}\right)^{-\frac{N+2}{2}} 2^{-(N+2)^{2}} \omega^{\left(1+\gamma^{+}\right) \frac{N+2}{2}}
$$

then $Y_{n} \rightarrow 0$ as $n \rightarrow \infty$. Taking

$$
\nu_{0} \equiv C\left(M, N, \gamma^{+}\right)^{-\frac{N+2}{2}} 2^{-(N+2)^{2}} \omega^{\left(1+\gamma^{+}\right) \frac{N+2}{2}}
$$

the above inequality is valid since it is no other than our hypothesis. Since $R_{n} \searrow \frac{R}{2}, k_{n} \searrow \frac{\omega}{4}$, and $Y_{n} \rightarrow 0$ as $n \rightarrow \infty$ implies that $A_{n} \rightarrow 0$ as $n \rightarrow \infty$, we obtain

that is,

$$
\left|(x, z) \in Q\left(\left(\frac{R}{2}\right)^{2}, \frac{R}{2}\right): \bar{u}_{\omega}(x, z) \leq \frac{\omega}{4}\right|=0,
$$

$$
u(x, t) \geq \frac{\omega}{4}, \quad \text { a.e. }(x, t) \in Q\left(a_{0}\left(\frac{R}{2}\right)^{2}, \frac{R}{2}\right) .
$$

The reduction of the oscillation of $u$ follows at once. Indeed, we have

Corollary 1. There exist constants $\nu_{0} \in(0,1)$, depending on the data and $\omega$, and $\sigma_{0} \in(0,1)$, such that if $(7)$ holds then

$$
\underset{Q\left(a_{0}\left(\frac{R}{2}\right)^{2}, \frac{R}{2}\right)}{\operatorname{ess} \text { osc }} u \leq \sigma_{0} \omega .
$$

Proof. From Proposition 2 we know that

$$
\underset{Q\left(a_{0}\left(\frac{R}{2}\right)^{2}, \frac{R}{2}\right)}{\operatorname{ess} \inf } u(x, t) \geq \frac{\omega}{4} .
$$

Thereby, since $\mu^{+} \geq \underset{Q\left(a_{0}\left(\frac{R}{2}\right)^{2}, \frac{R}{2}\right)}{\operatorname{ess} \sup } u$,

$$
\underset{Q\left(a_{0}\left(\frac{R}{2}\right)^{2}, \frac{R}{2}\right)}{\operatorname{ess}} u \leq \mu^{+}-\frac{\omega}{4}=\left(1-\frac{1}{4}\right) \omega \equiv \sigma_{0} \omega .
$$




\section{The alternative}

Now we assume that (7) does not hold, so therefore (8) is in force. We will show that, in this case, we can get a result similar to (12). Remember that $\nu_{0}$ was already determined and is given by (11).

Lemma 1. Assume that (8) holds true. There exists a time level

$$
t_{0} \in\left[-a_{0} R^{2},-\frac{\nu_{0}}{2} a_{0} R^{2}\right]
$$

such that

$$
\left|x \in K_{R}: u\left(x, t_{0}\right)>\mu^{+}-\frac{\omega}{2}\right|<\left(\frac{1-\nu_{0}}{1-\frac{\nu_{0}}{2}}\right)\left|K_{R}\right| .
$$

Proof. In fact, if not then

$$
\begin{aligned}
\mid(x, t) \in & Q\left(a_{0} R^{2}, R\right): u(x, t)>\mu^{+}-\frac{\omega}{2} \mid \\
& \geq \int_{-a_{0} R^{2}}^{-\frac{\nu_{0}}{2} a_{0} R^{2}}\left|x \in K_{R}: u(x, t)>\mu^{+}-\frac{\omega}{2}\right| d t \\
& \geq\left(\frac{1-\nu_{0}}{1-\frac{\nu_{0}}{2}}\right)\left|K_{R}\right|\left(1-\frac{\nu_{0}}{2}\right) a_{0} R^{2} \\
& =\left(1-\nu_{0}\right)\left|Q\left(a_{0} R^{2}, R\right)\right|
\end{aligned}
$$

contradicting (8).

Accordingly, at time level $t_{0}$, the portion of the cube $K_{R}$ where $u(x)$ is close to its supremum is small. In what follows we will show that the same happens for all time levels near the top of the cylinder $Q\left(a_{0} R^{2}, R\right)$.

Lemma 2. There exists $1<s_{1} \in \mathbf{N}$, depending on the data and $\omega$, such that, for all $t \in\left(t_{0}, 0\right)$,

$$
\left|x \in K_{R}: u(x, t)>\mu^{+}-\frac{\omega}{2^{s_{1}}}\right|<\left(1-\left(\frac{\nu_{0}}{2}\right)^{2}\right)\left|K_{R}\right| .
$$

Proof. Consider the cylinder $Q\left(t_{0}, R\right)$ and the level $k=\mu^{+}-\frac{\omega}{2}$. Define

$$
u-k \leq H_{k}^{+} \equiv \operatorname{ess}_{Q\left(t_{0}, R\right)}(u-k)_{+} \leq \frac{\omega}{2},
$$


which we assume to be strictly positive (otherwise there will be nothing to prove). Select $n_{*} \in \mathbf{N}$ big enough so that $0<c=\frac{\omega}{2^{n_{*}+1}}<H_{k}^{+}$. Then the logarithmic function $\psi^{+}$given by

$$
\psi^{+}=\left\{\begin{array}{ccc}
\ln \left(\frac{H_{k}^{+}}{H_{k}^{+}-u+k+c}\right) & \text { if } & u>k+c \\
0 & \text { if } & u \leq k+c
\end{array}\right.
$$

is well defined and satisfies the inequalities

$$
\psi^{+} \leq \ln \left(2^{n_{*}}\right)=n_{*} \ln 2, \quad \text { since } \quad \frac{H_{k}^{+}}{H_{k}^{+}-u+k+c} \leq \frac{H_{k}^{+}}{c} \leq \frac{\frac{\omega}{2}}{\frac{\omega}{2^{n_{*}+1}}}=2^{n_{*}}
$$

and, for $u \neq k+c$,

$$
0 \leq\left(\psi^{+}\right)^{\prime} \leq \frac{1}{H_{k}^{+}-u+k+c} \leq \frac{1}{c}=\frac{2^{n_{*}+1}}{\omega}
$$

and

$$
\left(\psi^{+}\right)^{\prime \prime}=\left[\left(\psi^{+}\right)^{\prime}\right]^{2} \geq 0
$$

In the weak formulation (3) [to be rigorous we should consider, as before, formulation (4), integrate and take the limit as $h \rightarrow 0$; to simplify we proceed formally at this stage] consider the integration over $K_{R} \times\left(t_{0}, t\right)$, with $t \in$ $\left(t_{0}, 0\right)$, and take $\phi=2 \psi^{+}\left(\psi^{+}\right)^{\prime} \xi^{2}$, where $x \rightarrow \xi(x)$ is a smooth cutoff function defined in $K_{R}$ and verifying

$$
\left\{\begin{array}{l}
0 \leq \xi \leq 1 \quad \text { in } \quad K_{R} \\
\xi \equiv 1 \quad \text { in } \quad K_{(1-\sigma) R}, \quad \text { for some } \sigma \in(0,1) \\
|\nabla \xi| \leq \frac{C}{\sigma R} .
\end{array}\right.
$$

Then, for all $t \in\left(t_{0}, 0\right)$,

$$
\begin{aligned}
0 & =\int_{t_{0}}^{t} \int_{K_{R}} \partial_{t} u 2 \psi^{+}\left(\psi^{+}\right)^{\prime} \xi^{2}+\int_{t_{0}}^{t} \int_{K_{R}} u^{\gamma(x, t)} \nabla u \cdot \nabla\left(2 \psi^{+}\left(\psi^{+}\right)^{\prime} \xi^{2}\right) \\
& =: J_{1}+J_{2} .
\end{aligned}
$$

The two integrals can the estimated as follows:

$$
J_{1}=\int_{K_{R} \times\{t\}}\left(\psi^{+}\right)^{2} \xi^{2}-\int_{K_{R} \times\left\{t_{0}\right\}}\left(\psi^{+}\right)^{2} \xi^{2}
$$




$$
\geq \int_{K_{R} \times\{t\}}\left(\psi^{+}\right)^{2} \xi^{2}-n_{*}^{2} \ln ^{2} 2\left(\frac{1-\nu_{0}}{1-\frac{\nu_{0}}{2}}\right)\left|K_{R}\right|,
$$

using the estimate for $\psi^{+}$and Lemma 1;

$$
\begin{aligned}
J_{2}= & \int_{t_{0}}^{t} \int_{K_{R}} u^{\gamma(x, t)}|\nabla u|^{2} 2\left(1+\psi^{+}\right)\left[\left(\psi^{+}\right)^{\prime}\right]^{2} \xi^{2} \\
& +2 \int_{t_{0}}^{t} \int_{K_{R}} u^{\gamma(x, t)} \nabla u \cdot \nabla \xi 2 \psi^{+}\left(\psi^{+}\right)^{\prime} \xi \\
\geq & -\int_{t_{0}}^{t} \int_{K_{R}} u^{\gamma(x, t)}|\nabla \xi|^{2} 2 \psi^{+},
\end{aligned}
$$

using Cauchy's inequality. Putting these estimates together, using the bounds for $|\nabla \xi|$ and $\psi^{+}$, and recalling what $t_{0}$ and $a_{0}$ are, we arrive at

$$
\begin{aligned}
\int_{K_{R} \times\{t\}}\left(\psi^{+}\right)^{2} \xi^{2} & \leq\left[n_{*}^{2} \ln ^{2} 2\left(\frac{1-\nu_{0}}{1-\frac{\nu_{0}}{2}}\right)+2 n_{*} \ln 2 \frac{C}{\sigma^{2} R^{2}}\left(-t_{0}\right)\right]\left|K_{R}\right| \\
& \leq\left[n_{*}^{2} \ln ^{2} 2\left(\frac{1-\nu_{0}}{1-\frac{\nu_{0}}{2}}\right)+2 n_{*} \ln 2 \frac{C}{\sigma^{2}} a_{0}\right]\left|K_{R}\right| \\
& \leq\left[n_{*}^{2} \ln ^{2} 2\left(\frac{1-\nu_{0}}{1-\frac{\nu_{0}}{2}}\right)+2 n_{*} \ln 2 \frac{C\left(\gamma^{+}\right)}{\sigma^{2} \omega^{\gamma^{+}}}\right]\left|K_{R}\right|
\end{aligned}
$$

valid for all $t \in\left(t_{0}, 0\right)$.

The left hand side is estimated from below considering integration over the smaller set

$$
S=\left\{x \in K_{(1-\sigma) R}: u(x, t)>\mu^{+}-\frac{\omega}{2^{n_{*}+1}}\right\} .
$$

On $S, \xi \equiv 1$ and $\psi^{+} \geq\left(n_{*}-1\right) \ln 2$, because

$$
\frac{H_{k}^{+}}{H_{k}^{+}-u+k+c} \geq \frac{H_{k}^{+}}{H_{k}^{+}-\frac{\omega}{2}+\frac{\omega}{2^{n_{*}}}}=\frac{H_{k}^{+}-\frac{\omega}{2}+\frac{\omega}{2}}{H_{k}^{+}-\frac{\omega}{2}+\frac{\omega}{2^{n_{*}}}} \geq 2^{n_{*}-1},
$$

since one has $H_{k}^{+}-\frac{\omega}{2} \leq 0$ and $\frac{\omega}{2}>\frac{\omega}{2^{n_{*}}}, \forall n_{*}>1$. Therefore, for all $t \in\left(t_{0}, 0\right)$,

$$
|S| \leq\left\{\left(\frac{n_{*}}{n_{*}-1}\right)^{2}\left(\frac{1-\nu_{0}}{1-\frac{\nu_{0}}{2}}\right)+\frac{C}{\sigma^{2} n_{*} \omega^{\gamma^{+}}}\right\}\left|K_{R}\right| .
$$

Consequently, for all $t \in\left(t_{0}, 0\right)$,

$$
\left|x \in K_{R}: u(x, t)>\mu^{+}-\frac{\omega}{2^{n_{*}+1}}\right| \leq|S|+N \sigma\left|K_{R}\right|
$$




$$
\leq\left\{\left(\frac{n_{*}}{n_{*}-1}\right)^{2}\left(\frac{1-\nu_{0}}{1-\frac{\nu_{0}}{2}}\right)+\frac{C}{\sigma^{2} n_{*} \omega^{\gamma^{+}}}+N \sigma\right\}\left|K_{R}\right| .
$$

The proof is complete once we choose $\sigma$ so small that $N \sigma \leq \frac{3}{8} \nu_{0}^{2}$, then $n_{*}$ so large that

$$
\frac{C}{n_{*} \sigma^{2} \omega^{\gamma^{+}}} \leq \frac{3}{8} \nu_{0}^{2} \quad \text { and } \quad\left(\frac{n_{*}}{n_{*}-1}\right)^{2} \leq\left(1-\frac{\nu_{0}}{2}\right)\left(1+\nu_{0}\right) \equiv \beta>1,
$$

and finally take $s_{1}=n_{*}+1$.

Remark 3. Note that, from the choice of $\sigma$, we get $\sigma<\frac{3}{8 N} \nu_{0}^{2}$ and from the two conditions on $n_{*}$ we obtain

$$
n_{*} \geq \max \left\{C \nu_{0}^{-6} \omega^{-\gamma^{+}} ; \frac{4}{\nu_{0}^{2}}+2\right\} .
$$

Clearly the number $s_{1}$ depends on the data as well as on $\omega$.

Recalling that $t_{0} \in\left[-a_{0} R^{2},-\frac{\nu_{0}}{2} a_{0} R^{2}\right]$, the previous lemma implies

Corollary 2. There exists $1<s_{1} \in \mathbf{N}$, depending on the data and $\omega$, such that, for all $t \in\left(-\frac{\nu_{0}}{2} a_{0} R^{2}, 0\right)$,

$$
\left|x \in K_{R}: u(x, t)>\mu^{+}-\frac{\omega}{2^{s_{1}}}\right|<\left(1-\left(\frac{\nu_{0}}{2}\right)^{2}\right)\left|K_{R}\right| .
$$

The conclusion of Corollary 2 will be employed to deduce that, within the cylinder $Q\left(\frac{\nu_{0}}{2} a_{0} R^{2}, R\right)$, the set where $u$ is close to its supremum is arbitrarily small.

Lemma 3. For all $\nu \in(0,1)$ there exists $s_{1}<s_{2} \in \mathbf{N}$, depending on the data and $\omega$, such that

$$
\left|(x, t) \in Q\left(\frac{\nu_{0}}{2} a_{0} R^{2}, R\right): u(x, t)>\mu^{+}-\frac{\omega}{2^{s_{2}}}\right| \leq \nu\left|Q\left(\frac{\nu_{0}}{2} a_{0} R^{2}, R\right)\right| .
$$

Proof. Consider the cylinder $Q\left(\nu_{0} a_{0} R^{2}, 2 R\right)$ and the levels $k=\mu^{+}-\frac{\omega}{2^{s}}$, for $s \geq s_{1}$. In order to obtain energy estimates for the functions $(u-k)_{+}$over 
this cylinder, in $(3)$ we take $\phi=(u-k)_{+} \xi^{2}$, where $0 \leq \xi \leq 1$ is a smooth cutoff function defined in $Q\left(\nu_{0} a_{0} R^{2}, 2 R\right)$ and satisfying

$$
\left\{\begin{array}{l}
\xi \equiv 0 \quad \text { on } \quad \partial_{p} Q\left(\nu_{0} a_{0} R^{2}, 2 R\right) \\
\xi \equiv 1 \quad \text { in } \quad Q\left(\frac{\nu_{0}}{2} a_{0} R^{2}, R\right) \\
|\nabla \xi| \leq \frac{1}{R} ; \quad 0 \leq \partial_{t} \xi \leq \frac{1}{\frac{\nu_{0}}{2} a_{0} R^{2}}
\end{array}\right.
$$

where $\partial_{p} Q\left(\nu_{0} a_{0} R^{2}, 2 R\right)$ denotes the parabolic boundary of $Q\left(\nu_{0} a_{0} R^{2}, 2 R\right)$. Then, for $t \in\left(-\nu_{0} a_{0} R^{2}, 0\right)$, we obtain (again formally)

$$
\int_{-\nu_{0} a_{0} R^{2}}^{t} \int_{K_{2 R}} \partial_{t} u(u-k)_{+} \xi^{2}+\int_{-\nu_{0} a_{0} R^{2}}^{t} \int_{K_{2 R}} u^{\gamma(x, t)} \nabla u \cdot \nabla\left((u-k)_{+} \xi^{2}\right)=0 .
$$

Now, since $(u-k)_{+} \leq \frac{\omega}{2^{s}}$,

$$
\begin{aligned}
\int_{-\nu_{0} a_{0} R^{2}}^{t} \int_{K_{2 R}} \partial_{t} u(u-k)_{+} \xi^{2}= & \frac{1}{2} \int_{K_{2 R} \times\{t\}}(u-k)_{+}^{2} \xi^{2} \\
& -\int_{-\nu_{0} a_{0} R^{2}}^{t} \int_{K_{2 R}}(u-k)_{+}^{2} \xi \partial_{t} \xi \\
\geq & -\left(\frac{\omega}{2^{s}}\right)^{2} \frac{1}{\frac{\nu_{0}}{2} a_{0} R^{2}} \int_{-\nu_{0} a_{0} R^{2}}^{t} \int_{K_{2 R}} \chi_{[u>k]}
\end{aligned}
$$

and

$$
\begin{gathered}
\int_{-\nu_{0} a_{0} R^{2}}^{t} \int_{K_{2 R}} u^{\gamma(x, t)} \nabla u \cdot \nabla\left((u-k)_{+} \xi^{2}\right) \\
\geq \frac{1}{2 a_{0}} \int_{-\nu_{0} a_{0} R^{2}}^{t} \int_{K_{2 R}}\left|\nabla(u-k)_{+}\right|^{2} \xi^{2}-2\left(\frac{\omega}{2^{s}}\right)^{2} \frac{1}{R^{2}} \int_{-\nu_{0} a_{0} R^{2}}^{t} \int_{K_{2 R}} \chi_{[u>k]},
\end{gathered}
$$

using Cauchy's inequality with $\epsilon=\frac{1}{4}$ and the fact that when $(u-k)_{+}$is not zero, then

$$
u>k=\mu^{+}-\frac{\omega}{2^{s}}>\mu^{+}-\frac{\omega}{2}=\frac{\omega}{2}>\frac{\omega}{4},
$$

since $s \geq s_{1}>1$, and therefore

$$
1 \geq u^{\gamma(x, t)} \geq\left(\frac{\omega}{4}\right)^{\gamma^{+}}=\frac{1}{a_{0}} .
$$


We then have

$$
\begin{gathered}
\frac{1}{2 a_{0}} \iint_{Q\left(\frac{\nu_{0}}{2} a_{0} R^{2}, R\right)}\left|\nabla(u-k)_{+}\right|^{2} \leq\left(\frac{\omega}{2^{s}}\right)^{2} \frac{1}{R^{2}}\left(\frac{1}{\frac{\nu_{0}}{2} a_{0}}+2\right) \iint_{Q\left(\nu_{0} a_{0} R^{2}, 2 R\right)} \chi_{[u>k]} \\
\leq\left(\frac{\omega}{2^{s}}\right)^{2} \frac{1}{R^{2}}\left(\frac{1}{\frac{\nu_{0}}{2} a_{0}}+2\right) 2^{N+1}\left|Q\left(\frac{\nu_{0}}{2} a_{0} R^{2}, R\right)\right|
\end{gathered}
$$

and consequently, multiplying by $2 a_{0}$,

$$
\begin{aligned}
\iint_{Q\left(\frac{\nu_{0}}{2} a_{0} R^{2}, R\right)}\left|\nabla(u-k)_{+}\right|^{2} & \leq\left(\frac{\omega}{2^{s}}\right)^{2} \frac{1}{R^{2}}\left(\frac{4}{\nu_{0}}+4 a_{0}\right) 2^{N+1}\left|Q\left(\frac{\nu_{0}}{2} a_{0} R^{2}, R\right)\right| \\
& =\frac{2^{N+3}}{\nu_{0}}\left(\frac{\omega}{2^{s}}\right)^{2} \frac{1}{R^{2}}\left(1+a_{0} \nu_{0}\right)\left|Q\left(\frac{\nu_{0}}{2} a_{0} R^{2}, R\right)\right| \\
& \leq \frac{C\left(M, N, \gamma^{+}\right)}{\omega^{\left(1+\gamma^{+}\right) \frac{(N+2)}{2}}}\left(\frac{\omega}{2^{s}}\right)^{2} \frac{1}{R^{2}}\left|Q\left(\frac{\nu_{0}}{2} a_{0} R^{2}, R\right)\right|
\end{aligned}
$$

recalling the definition of $\nu_{0}$ given by (11).

Now we consider the levels $l=\mu^{+}-\frac{\omega}{2^{s+1}}>k=\mu^{+}-\frac{\omega}{2^{s}}, s=s_{1}, \ldots, s_{2}-1$, and define, for $t \in\left(-\frac{\nu_{0}}{2} a_{0} R^{2}, 0\right)$,

$$
A_{s}(t) \equiv\left\{x \in K_{R}: u(x, t)>\mu^{+}-\frac{\omega}{2^{s}}\right\}
$$

and

$$
A_{s} \equiv \int_{-\frac{\nu_{0}}{2} a_{0} R^{2}}^{0}\left|A_{s}(t)\right| d t .
$$

Using Lemma 2.2 of $[4$, page 5] applied to the function $u(\cdot, t)$ for all times $t \in\left(-\frac{\nu_{0}}{2} a_{0} R^{2}, 0\right)$, we get

$$
\left(\frac{\omega}{2^{s+1}}\right)\left|A_{s+1}(t)\right| \leq C(N) \frac{R^{N+1}}{\left|K_{R} \backslash A_{s}(t)\right|} \int_{[k<u<l]}|\nabla u| .
$$

Since $\mu^{+}-\frac{\omega}{2^{s}} \geq \mu^{+}-\frac{\omega}{2^{s_{1}}}$, for $s \geq s_{1}$,

$$
\left|A_{s}(t)\right| \leq\left|A_{s_{1}}(t)\right|<\left(1-\left(\frac{\nu_{0}}{2}\right)^{2}\right)\left|K_{R}\right|, \quad \forall t \in\left(-\frac{\nu_{0}}{2} a_{0} R^{2}, 0\right),
$$

by virtue of (16). Then, for all $t \in\left(-\frac{\nu_{0}}{2} a_{0} R^{2}, 0\right)$,

$$
\left(\frac{\omega}{2^{s+1}}\right)\left|A_{s+1}(t)\right| \leq \frac{C(N)}{\nu_{0}^{2}} R \int_{[k<u<l]}|\nabla u|
$$


and finally, integrating in time over $\left(-\frac{\nu_{0}}{2} a_{0} R^{2}, 0\right)$ and using Hölder's inequality, we arrive at

$$
\begin{gathered}
\left(\frac{\omega}{2^{s+1}}\right) A_{s+1} \leq \frac{C(N)}{\nu_{0}^{2}} R \int_{-\frac{\nu_{0}}{2} a_{0} R^{2}}^{0} \int_{[k<u<l]}|\nabla u| \\
\leq \frac{C(N)}{\nu_{0}^{2}} R\left(\iint_{Q\left(\frac{\nu_{0}}{2} a_{0} R^{2}, R\right)}\left|\nabla(u-k)_{+}\right|^{2}\right)^{\frac{1}{2}}\left|A_{s} \backslash A_{s+1}\right|^{\frac{1}{2}} .
\end{gathered}
$$

According to the previous energy estimates we get, for $s=s_{1}, \ldots, s_{2}-1$,

$$
A_{s+1}^{2} \leq \frac{C\left(M, N, \gamma^{+}\right)}{\omega^{\frac{5}{2}\left(1+\gamma^{+}\right)(N+2)}}\left|Q\left(\frac{\nu_{0}}{2} a_{0} R^{2}, R\right)\right|\left|A_{s} \backslash A_{s+1}\right|,
$$

and we then add these inequalities for $s=s_{1}, \ldots, s_{2}-1$.

Since $\mu^{+}-\frac{\omega}{2^{s+1}} \leq \mu^{+}-\frac{\omega}{2^{s_{2}}}, A_{s+1} \geq A_{s_{2}}$, and therefore

$$
\sum_{s=s_{1}}^{s_{2}-1} A_{s+1}^{2} \geq\left(s_{2}-s_{1}\right) A_{s_{2}}^{2} \text {. }
$$

Note also that $\sum_{s=s_{1}}^{s_{2}-1}\left|A_{s} \backslash A_{s+1}\right| \leq\left|Q\left(\frac{\nu_{0}}{2} a_{0} R^{2}, R\right)\right|$. Collecting results, we arrive at

$$
A_{s_{2}}^{2} \leq \frac{C\left(M, N, \gamma^{+}\right)}{\omega^{\frac{5}{2}\left(1+\gamma^{+}\right)(N+2)}\left(s_{2}-s_{1}\right)}\left|Q\left(\frac{\nu_{0}}{2} a_{0} R^{2}, R\right)\right|^{2}
$$

and the proof is complete if we choose $s_{1}<s_{2} \in \mathbf{N}$ sufficiently large so that

$$
\frac{C\left(M, N, \gamma^{+}\right)}{\omega^{\frac{5}{2}\left(1+\gamma^{+}\right)(N+2)}\left(s_{2}-s_{1}\right)} \leq \nu^{2}
$$

Remark 4. It is clear from the indicated choice of $s_{2}$ that it depends on $\omega$.

Lemma 4. The number $\nu \in(0,1)$ can be chosen (and consequently, so can $\left.s_{2}\right)$ such that

$$
u(x, t) \leq \mu^{+}-\frac{\omega}{2^{s_{2}+1}}, \quad \text { a.e. } \quad(x, t) \in Q\left(\frac{\nu_{0}}{2} a_{0}\left(\frac{R}{2}\right)^{2}, \frac{R}{2}\right) .
$$


Proof. Define two sequences of positive real numbers

$$
R_{n}=\frac{R}{2}+\frac{R}{2^{n+1}}, \quad k_{n}=\mu^{+}-\frac{\omega}{2^{s_{2}+1}}-\frac{\omega}{2^{s_{2}+1+n}}, \quad n=0,1,2, \ldots
$$

and construct the family of nested and shrinking cylinders

$$
Q_{n}=Q\left(\frac{\nu_{0}}{2} a_{0} R_{n}^{2}, R_{n}\right)
$$

Consider the function $u_{\omega}=\min \left\{u, \mu^{+}-\frac{\omega}{2^{s_{2}+1}}\right\}$ and, in the weak formulation (3), take $\phi=\left(u_{\omega}-k_{n}\right)_{+} \xi_{n}^{2}$, where $0 \leq \xi_{n} \leq 1$ are smooth cutoff functions defined in $Q_{n}$ and verifying

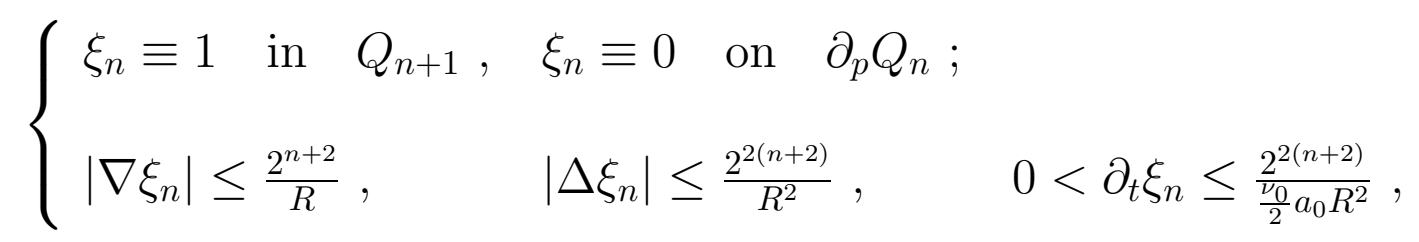

where $\partial_{p} Q_{n}$ denotes the parabolic boundary of $Q_{n}$.

Then, for $t \in\left(-\frac{\nu_{0}}{2} a_{0} R_{n}^{2}, 0\right)$, we have formally (again, to be rigorous we would have to argue with the Steklov averages and pass to the limit in $h$ )

$$
\begin{gathered}
0=\int_{-\frac{\nu_{0}}{2} a_{0} R_{n}^{2}}^{t} \int_{K_{R_{n}}} \partial_{t} u\left(\left(u_{\omega}-k_{n}\right)_{+} \xi_{n}^{2}\right) \\
+\int_{-\frac{\nu_{0}}{2} a_{0} R_{n}^{2}}^{t} \int_{K_{R_{n}}} u^{\gamma(x, t)} \nabla u \cdot \nabla\left(\left(u_{\omega}-k_{n}\right)_{+} \xi_{n}^{2}\right):=I_{1}+I_{2} .
\end{gathered}
$$

Observe that

$$
\begin{gathered}
I_{1}=\int_{-\frac{\nu_{0}}{2} a_{0} R_{n}^{2}}^{t} \int_{K_{R_{n}}} \partial_{t} u\left(\left(u_{\omega}-k_{n}\right)+\xi_{n}^{2}\right) \chi_{\left[u_{\omega}=u\right]} \\
+\int_{-\frac{\nu_{0}}{2} a_{0} R_{n}^{2}}^{t} \int_{K_{R_{n}}} \partial_{t} u\left(\left(u_{\omega}-k_{n}\right)_{+} \xi_{n}^{2}\right) \chi_{\left[u_{\omega}=\mu^{+}-\frac{\omega}{2^{s_{2}+1}}\right]} \\
=\frac{1}{2} \int_{K_{R_{n}} \times\{t\}}\left(u_{\omega}-k_{n}\right)_{+}^{2} \xi_{n}^{2}-\int_{-\frac{\nu_{0}}{2} a_{0} R_{n}^{2}}^{t}\left(u_{K_{R_{n}}}\left(u_{\omega}-k_{n}\right)_{+}^{2} \partial_{t} \xi_{n} \xi_{n}\right. \\
+\left(\frac{\omega}{2^{s_{2}+1+n}}\right) \int_{-\frac{\nu_{0}}{2} a_{0} R_{n}^{2}}^{t} \int_{K_{R_{n}}} \partial_{t}\left(u-\left(\mu^{+}-\frac{\omega}{2^{s_{2}+1}}\right)\right)_{+} \xi_{n}^{2} \\
\geq \frac{1}{2} \int_{K_{R_{n}} \times\{t\}}\left(u_{\omega}-k_{n}\right)_{+}^{2} \xi_{n}^{2}-3\left(\frac{\omega}{2^{s_{2}+1}}\right)^{2} \frac{2^{2(n+2)}}{\frac{\nu_{0}}{2} a_{0} R^{2}} \int_{-\frac{\nu_{0}}{2} a_{0} R_{n}^{2}}^{t} \int_{K_{R_{n}}} \chi_{\left[u_{\omega}>k_{n}\right]},
\end{gathered}
$$


arguing as in the proof of Proposition 2 (with the obvious changes). Concerning $I_{2}$, we have

$$
\begin{aligned}
I_{2}= & \int_{-\frac{\nu_{0}}{2} a_{0} R_{n}^{2}}^{t} \int_{K_{R_{n}}} u^{\gamma(x, t)} \nabla u \cdot \nabla\left(\left(u_{\omega}-k_{n}\right)_{+} \xi_{n}^{2}\right) \chi_{\left[u_{\omega}=u\right]} \\
& +\int_{-\frac{\nu_{0}}{2} a_{0} R_{n}^{2}}^{t} \int_{K_{R_{n}}} u^{\gamma(x, t)} \nabla u \cdot \nabla\left(\left(u_{\omega}-k_{n}\right)_{+} \xi_{n}^{2}\right) \chi_{\left[u_{\omega}=\mu^{+}-\frac{\omega}{2^{s_{2}+1}}\right]} \\
\geq & \frac{1}{2} \int_{-\frac{\nu_{0}}{2} a_{0} R_{n}^{2}}^{t} \int_{K_{R_{n}}} u_{\omega}^{\gamma(x, t)}\left|\nabla\left(u_{\omega}-k_{n}\right)_{+}\right|^{2} \xi_{n}^{2} \\
& -2 \int_{-\frac{\nu_{0}}{2} a_{0} R_{n}^{2}}^{t} \int_{K_{R_{n}}} u_{\omega}^{\gamma(x, t)}\left(u_{\omega}-k_{n}\right)_{+}^{2}\left|\nabla \xi_{n}\right|^{2} \\
& -2\left(\frac{\omega}{2^{s_{2}+1}}\right) \int_{-\frac{\nu_{0}}{2} a_{0} R_{n}^{2}}^{t} \int_{K_{R_{n}}}\left(\int_{\mu^{+}-\frac{\omega}{2^{s_{2}+1}}}^{u} s^{\gamma(x, t)} d s\right) \\
& -\left(\frac{\omega}{2^{s_{2}+1}}\right) \int_{-\frac{\nu_{0}}{2} a_{0} R_{n}^{2}}^{t} \int_{\left.\left.K_{R_{n}}|+| \nabla \xi_{n}\right|^{2}\right) \chi_{\left[u \geq \mu^{+}-\frac{\omega}{2^{s_{2}+1}}\right]}\left(\int_{\mu^{+}-\frac{\omega}{2^{s_{2}+1}}}^{u} s^{\gamma(x, t)}(-\ln s) d s\right)}^{\left(|\nabla \gamma|^{2}+\left|\nabla \xi_{n}\right|^{2}\right) \chi_{\left[u \geq \mu^{+}-\frac{\omega}{2^{s_{2}+1}}\right]},}
\end{aligned}
$$

using Cauchy's inequality and integration by parts.

Noting that, when $k_{n}<u=u_{\omega}<\mu^{+}-\frac{\omega}{2^{s_{2}+1}}<1$,

$$
\frac{\omega}{4}<\frac{\omega}{2}=\mu^{+}-\frac{\omega}{2}<\mu^{+}-\frac{\omega}{2^{s_{1}}}<\mu^{+}-\frac{\omega}{2^{s_{2}}}<k_{n}<u_{\omega} \leq 1
$$

(since $1<s_{1}<s_{2}$ ) and then

$$
\frac{1}{a_{0}} \leq u_{\omega}^{\gamma(x, t)} \leq 1 \quad \text { and } \quad\left(u_{\omega}-k_{n}\right)_{+} \leq \frac{\omega}{2^{s_{2}+1}} ;
$$

when $u \geq \mu^{+}-\frac{\omega}{2^{s_{2}+1}}$

$$
u_{\omega}=\mu^{+}-\frac{\omega}{2^{s_{2}+1}} \geq k_{n}
$$

and also that

$$
\int_{\mu^{+}-\frac{\omega}{2^{s_{2}+1}}}^{u} s^{\gamma(x, t)} d s \leq \frac{\omega}{2^{s_{2}+1}}
$$


and

$$
\begin{aligned}
\int_{\mu^{+}-\frac{\omega}{2^{s_{2}+1}}}^{u} s^{\gamma(x, t)}(-\ln s) d s & \leq \int_{\mu^{+}-\frac{\omega}{2^{s_{2}+1}}}^{u}(-\ln s) d s \\
& \leq\left(\frac{\omega}{2^{s_{2}+1}}\right)\left(1-\ln \left(\mu^{+}-\frac{\omega}{2^{s_{2}+1}}\right)\right) \\
& \leq\left(\frac{\omega}{2^{s_{2}+1}}\right) \frac{2}{\omega}
\end{aligned}
$$

(since, as $s_{2}>s_{1}>1, \mu^{+}-\frac{\omega}{2^{s_{2}+1}}>\mu^{+}-\frac{\omega}{2^{s_{1}}}>\mu^{+}-\frac{\omega}{2}=\frac{\omega}{2}$ ), we obtain, with a reasoning similar to the one employed in the proof of Proposition 2, the estimate

$$
\begin{gathered}
\sup _{-\frac{\nu_{0}}{2} a_{0} R_{n}^{2}<t<0} \int_{K_{R_{n}} \times\{t\}}\left(u_{\omega}-k_{n}\right)_{+}^{2} \xi_{n}^{2}+\frac{1}{a_{0}} \iint_{Q_{n}}\left|\nabla\left(u_{\omega}-k_{n}\right)_{+}\right|^{2} \xi_{n}^{2} \\
\leq\left(\frac{\omega}{2^{s_{2}+1}}\right)^{2} \frac{2^{2(n+2)}}{R^{2}}\left\{\frac{6}{\frac{\nu_{0}}{2} a_{0}}+\frac{C(M)}{\omega}\right\} \iint_{Q_{n}} \chi_{\left[u_{\omega} \geq k_{n}\right]} .
\end{gathered}
$$

Introducing the change of variables

$$
z=\frac{t}{\frac{\nu_{0}}{2} a_{0}}
$$

and defining the new functions

$$
\bar{u}_{\omega}(x, z)=u_{\omega}\left(x, \frac{\nu_{0}}{2} a_{0} z\right), \quad \bar{\xi}_{n}(x, z)=\xi_{n}\left(x, \frac{\nu_{0}}{2} a_{0} z\right),
$$

the previous estimate reads

$$
\begin{gathered}
\sup _{-R_{n}^{2}<z<0} \int_{K_{R_{n}} \times\{z\}}\left(\bar{u}_{\omega}-k_{n}\right)_{+}^{2} \bar{\xi}_{n}^{2}+\frac{\nu_{0}}{2} \iint_{Q\left(R_{n}^{2}, R_{n}\right)}\left|\nabla\left(\bar{u}_{\omega}-k_{n}\right)_{+}\right|^{2} \bar{\xi}_{n}^{2} \\
\leq\left(\frac{\omega}{2^{s_{2}+1}}\right)^{2} \frac{2^{2(n+2)}}{R^{2}}\left\{6+\frac{C(M)}{\omega} \nu_{0} a_{0}\right\} \iint_{Q\left(R_{n}^{2}, R_{n}\right)} \chi_{\left[\bar{u}_{\omega} \geq k_{n}\right]} .
\end{gathered}
$$

Multiplying the above estimate by $\frac{2}{\nu_{0}} \geq 1$, we arrive at

$$
\begin{aligned}
\left\|\left(\bar{u}_{\omega}-k_{n}\right)_{+}\right\|_{V^{2}\left(Q\left(R_{n+1}^{2}, R_{n+1}\right)\right)}^{2} & \leq C(M)\left(\frac{\omega}{2^{s_{2}+1}}\right)^{2} \frac{2^{2(n+2)}}{R^{2}}\left\{\frac{1}{\nu_{0}}+\frac{a_{0}}{\omega}\right\} A_{n} \\
& \leq \frac{C\left(M, N, \gamma^{+}\right)}{\omega^{\left(1+\gamma^{+}\right)\left(\frac{N+2}{2}\right)}}\left(\frac{\omega}{2^{s_{2}+1}}\right)^{2} \frac{2^{2(n+2)}}{R^{2}} A_{n}
\end{aligned}
$$


where $A_{n}$ is defined as

$$
A_{n} \equiv \int_{-R_{n}^{2}}^{0} \int_{K_{R_{n}}} \chi_{\left[\bar{u}_{\omega} \geq k_{n}\right]} .
$$

Observing that, on the one hand

$$
\iint_{Q\left(R_{n+1}^{2}, R_{n+1}\right)}\left(\bar{u}_{\omega}-k_{n}\right)_{+}^{2} \geq\left(k_{n+1}-k_{n}\right)^{2} A_{n+1}=\left(\frac{\omega}{2^{s_{2}+1}}\right)^{2} \frac{1}{2^{2(n+1)}} A_{n+1}
$$

and, on the other hand, using Corollary 3.1 of [4, page 9],

$$
\iint_{Q\left(R_{n+1}^{2}, R_{n+1}\right)}\left(\bar{u}_{\omega}-k_{n}\right)_{+}^{2} \leq C(N) A_{n}^{\frac{2}{N+2}}\left\|\left(\bar{u}-k_{n}\right)_{+}\right\|_{V^{2}\left(Q\left(R_{n+1}^{2}, R_{n+1}\right)\right)}^{2},
$$

we obtain

$$
A_{n+1} \leq \frac{C\left(M, N, \gamma^{+}\right)}{\omega^{\left(1+\gamma^{+}\right)\left(\frac{N+2}{2}\right)}} \frac{2^{4 n}}{R^{2}} A_{n}^{1+\frac{2}{N+2}}
$$

Defining $Y_{n} \equiv \frac{A_{n}}{\left|Q\left(R_{n}^{2}, R_{n}\right)\right|}$ and noting that $\frac{\left|Q\left(R_{n}^{2}, R_{n}\right)\right|^{1+\frac{2}{N+2}}}{\left|Q\left(R_{n+1}^{2}, R_{n+1}\right)\right|} \leq 2^{N+4} R^{2}$, we get the algebraic inequality

$$
Y_{n+1} \leq \frac{C\left(M, N, \gamma^{+}\right)}{\omega^{\left(1+\gamma^{+}\right)\left(\frac{N+2}{2}\right)}} 2^{4 n} Y_{n}^{1+\frac{2}{N+2}} .
$$

Using a well known result on the fast geometric convergence of sequences, namely Lemma 4.1 of [4, page 12], the result is proved if we can assure that

$$
\begin{aligned}
Y_{0} & \leq C\left(M, N, \gamma^{+}\right)^{-\frac{N+2}{2}} \omega^{\left(1+\gamma^{+}\right) \frac{(N+2)^{2}}{4}} 2^{-(N+2)^{2}} \\
& =C\left(M, N, \gamma^{+}\right) \omega^{\left(1+\gamma^{+}\right) \frac{(N+2)^{2}}{4}} \equiv \nu .
\end{aligned}
$$

For this value of $\nu$, by Lemma 3 , there exists $s_{1}<s_{2} \in \mathbf{N}$ such that

$$
\frac{\left|(x, z) \in Q\left(R^{2}, R\right): \bar{u}(x, z)>\mu^{+}-\frac{\omega}{2^{s_{2}}}\right|}{\left|Q\left(R^{2}, R\right)\right|} \leq \nu
$$

which implies $Y_{0} \leq \nu$. Then we can conclude that $Y_{n} \rightarrow 0$ when $n \rightarrow \infty$, and the result follows.

Proposition 3. There exist positive numbers $\nu_{0}, \sigma_{1} \in(0,1)$, depending on the data and on $\omega$, such that, if (8) holds true then

$$
\underset{Q\left(\frac{\nu_{0}}{2} a_{0}\left(\frac{R}{2}\right)^{2}, \frac{R}{2}\right)}{\operatorname{ess}} u \leq \sigma_{1} \omega .
$$


Proof. The proof is trivial and similar to the proof of Corollary 1. We have $\sigma_{1}=1-\frac{1}{2^{s_{2}+1}}$.

Proof of Proposition 1. Recalling the conclusions of Corollary 1 and Proposition 3, we take

$$
\begin{gathered}
\sigma=\max \left\{\sigma_{0}, \sigma_{1}\right\}=\sigma_{1}, \\
\text { since } \sigma_{0}=1-\frac{1}{4}<1-\frac{1}{2^{s_{2}+1}}=\sigma_{1}, \text { because } s_{2}>1 . \text { As } \nu_{0} \in(0,1) \\
Q\left(\frac{\nu_{0}}{2} a_{0}\left(\frac{R}{2}\right)^{2}, \frac{R}{2}\right) \subset Q\left(a_{0}\left(\frac{R}{2}\right)^{2}, \frac{R}{2}\right)
\end{gathered}
$$

and the result follows.

\section{References}

[1] S. Antontsev and S. Shmarev, A model porous medium equation with variable exponent of nonlinearity: existence, uniqueness and localization properties of solutions, Nonlinear Anal. 60 (2005), 515-545.

[2] L. Caffarelli and A. Friedman, Continuity of the density of a gas flow in a porous medium, Trans. Amer. Math. Soc. 252 (1979), 99-113.

[3] E. DiBenedetto, Continuity of weak solutions to a general porous medium equation, Indiana Univ. Math. J. 32 (1983), 83-118.

[4] _ Degenerate parabolic equations, Springer-Verlag, New York, 1993.

[5] E. DiBenedetto, J.M. Urbano, and V. Vespri, Current issues on singular and degenerate evolution equations, Handbook of Differential Equations, Evolutionary Equations, Vol. 1 (C. Dafermos and E. Feireisl, eds.), Elsevier, 2004, pp. 169-286.

[6] E. Henriques and J.M. Urbano, On the doubly singular equation $\gamma(u)_{t}=\Delta_{p} u$, Comm. Partial Differential Equations (to appear).

[7] M. Rǔžička, Electrorheological fluids: modelling and mathematical theory, Lecture Notes in Mathematics, vol. 1748, Springer-Verlag, Berlin, 2000.

[8] M.M. Porzio and V. Vespri, Hölder estimates for local solutions of some doubly nonlinear degenerate parabolic equations, J. Differential Equations 103 (1993), 146-178.

[9] J.M. Urbano, Continuous solutions for a degenerate free boundary problem, Ann. Mat. Pura Appl. 178 (2000), 195-224.

[10] _ Hölder continuity of local weak solutions for parabolic equations exhibiting two degeneracies, Adv. Differential Equations 6 (2001), 327-358.

EURICA HENRIQUeS

Departamento de Matemática, Utad, 5000-911 Vila Real, Portugal

E-mail address: eurica@utad.pt

José Miguel Urbano

Departamento de Matemática, Universidade de Coimbra, 3001-454 Coimbra, Portugal

E-mail address: jmurb@mat.uc.pt 